\title{
TWO LABELED EDTMP RADIOPHARMACEUTICALS WITH Sm-153 AND Lu-177 FOR HUMAN BONE RADIOTHERAPY
}

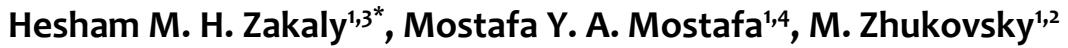 \\ ${ }^{1}$ Ural Federal University, Yekaterinburg, Russia \\ ${ }^{2}$ Russia Institute of Industrial Ecology UB RAS, Ekaterinburg, Russia \\ 3Al-Azhar University, Faculty of Science, Physics Department, Assuit, Egypt \\ ${ }^{4}$ Minia University, Faculty of Science, Department of Physics, El-Minia, Egypt
}

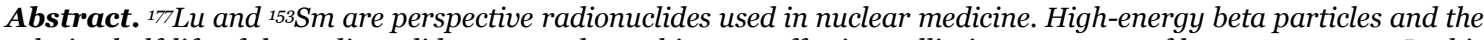
relative half-life of the radionuclides are used to achieve an effective palliative treatment of bone metastases. In this paper, the effect of the drug carrier EDTMP (i.e. ethylene diamine tetramethylene phosphonate) on the ionic form of

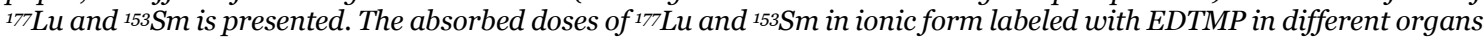
and tissues are determined by IDAC-Dose 2.1 (Internal Dose Assessment by Computer) software and WinAct software which is used to calculate cumulative activity. ${ }^{177} \mathrm{Lu}$ and ${ }^{153 \mathrm{Sm}}$ are lanthanide radionuclides which actively accumulate in the liver and bones when used in ionic form. In the case of labeling with EDTMP, the distribution and elimination of the drug occur according to the kinetics of the carrier, EDTMP. The use of an osteotropic complex (drugs attracted to and targeting bones) allows creating a large dose in the pathological areas and minimizes the damage of healthy organs

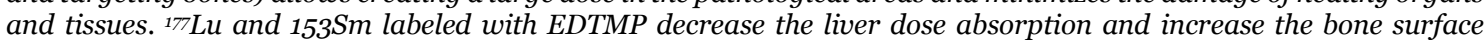
absorption for a more effective treatment and minimizing side effects. The effective dose per administered activity is

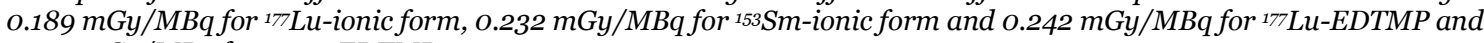
$0.139 \mathrm{mGy} / \mathrm{MBq}$ for ${ }^{153} \mathrm{sm}-\mathrm{EDTMP}$.
\end{abstract}

Keywords: Radiopharmaceutical, Sm-153, Lu-177, EDTMP

\section{INTRODUCTION}

Radionuclide therapy (RNT), employing radiopharmaceuticals labeled with $\beta^{-}$conversion electron-emitting radionuclides, is effectively utilized for bone pain palliation, thus providing significant improvement in the quality of life of patients suffering from pain resulting from secondary skeletal metastases. The major challenge in developing effective agents for the palliative treatment of bone pain arising from skeletal metastasis is to ensure the delivery of an adequate dose of ionizing radiation at the site of the skeletal lesion with minimum radiation-induced bone marrow suppression. These in vivo features are governed by the tissue penetration range and, hence, on the energies of the $\beta^{-}$particles of the radionuclides used in the radiopharmaceutical preparations $[1,2]$.

Designing ideal radiopharmaceuticals for use as bone pain palliatives requires the use of a moderate energy $\beta^{-}$emitter as a radionuclide and a suitable polyaminophosphonic acid as a carrier molecule. Owing to its suitable decay characteristics $[\mathrm{T} 1 / 2=6.73 \mathrm{~d}$, $\mathrm{E} \beta(\max )=497 \mathrm{keV}, \mathrm{E} \gamma=113 \mathrm{keV}$ (6.4\%), $208 \mathrm{keV}$ (11\%)] as well as the feasibility of large-scale production inadequate specific activity and radionuclidic purity using a moderate flux reactor, ${ }^{177} \mathrm{Lu}$ could be considered as a promising radionuclide for palliative care in painful bone metastases. Therefore, the present study is oriented toward the comparison of the ${ }^{177} \mathrm{Lu}$ complex of ethylene diamine tetramethylene phosphonic acid

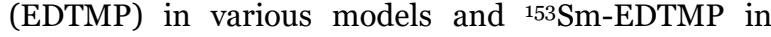
therapy [1].

${ }^{153} \mathrm{Sm}$ is a radionuclide emitting beta radiation with an average energy of $0.223 \mathrm{MeV}$ and accompanying gamma radiation with an energy of $103 \mathrm{Kev}$ (yield 29\%). The half-life of ${ }^{153 \mathrm{Sm}}$ is 46.3 hours, which imposes territorial restrictions. That is, the production of the radionuclide, the preparation of the drug and therapy should be carried out in a very short time. In North America, the drug ${ }^{153 \mathrm{Sm}-E D T M P}$ (ethylene diamine tetramethylene phosphonate) has been available for therapy since 1997 [2]. Since the use of this drug is possible with any kind of cancer which is accompanied by bone metastases, the potential market for radiopharmaceuticals is very large. ${ }^{153 \mathrm{Sm} \text {-oxabiphore is }}$ a drug used presently in Russia for the treatment of bone lesions - similar to the foreign ${ }^{153} \mathrm{Sm}$-EDTMP. This complex is concentrated in the skeleton in proportion to osteoblastic activity. Pathological foci, where the accumulation is intense, can be visualized using the gamma camera, which enables the scintigraphy of a patient and monitoring of the treatment process. The drug is very quickly excreted from the blood. 0.5-3 hours after intravenous injection only $1 \%$ of the drug remains in the blood. It is excreted from the urine almost completely after 6 hours [3-5]. The behavior of the whole drug in the body is affected by the nature of the ligand distribution, and the

\footnotetext{
*h.m.zakaly@gmail.com
} 
radionuclide in the complex serves either for the treatment of the disease or for diagnosis. The distribution of the ligand EDTMP is identical to the distribution of other complexes, isotropic to the bone tissue, for example methylene diphosphonate (MDP) $[2,3,6]$. Nowadays, methylene diphosphonate labeled with radionuclide $99 \mathrm{mTc}$ is used for the diagnosis of bone anomalies. Due to the gamma radiation emitted by the 99m Tc radionuclide, health care professionals can assess bone metabolism in detail and track where the drug accumulates the most, allowing for the therapy to be planned if necessary.

The purpose of this paper is to assess and examine the effect of the drug carrier EDTMP (i.e. ethylene diamine tetramethylene phosphonate) on the ionic form of ${ }^{177} \mathrm{Lu}$ and ${ }^{153 \mathrm{Sm}}$. Even in ionic form, the distribution of ${ }^{177} \mathrm{Lu}$ is better than the distribution of ${ }^{153 \mathrm{Sm}}$ being more absorbed in the bone surface, red bone marrow, and kidney with low absorption in life.

\section{MATERIALS AND METHODS}

The specialized package WinAct is developed in Oakridge laboratory [7] and it is intended for the assessment of the dynamics of the behavior of radionuclides in an organism. A biokinetic model of the drug EDTMP taking into account the conversion is presented in Figure 1. This model can be used for EDTMP labeled with ${ }^{177} \mathrm{Lu}$ or ${ }^{153 \mathrm{Sm}}$. Each rectangle shown in the diagram, Figure 1, corresponds to a linear differential equation of the first order, which is included in the general system of equations. Writing and solving such a system of equations for ${ }^{177} \mathrm{Lu}$ and ${ }^{153} \mathrm{Sm}$ takes a long time. The WinAct package is a specialized package for solving such systems [8,9].

The initial text block in the file is a comment block. For structuring the original design of the file into parts special words-separators are used. These words are the delimiters and are always typed in all uppercase letters. The activity distribution for ${ }^{177 \mathrm{Lu} \text { and }}{ }^{153 \mathrm{Sm}}$ in ionic

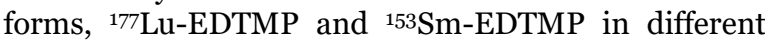
organs based on the model in Figure 1 are calculated as shown in Figure 2. The calculation was prepared for the diagnostic pathological area of bone tissue at a patient. The values of the transition factors of the substance from blood to organs are determined on the basis of a number of studies, converted from biokinetics for mice according to human anatomy $[1,8,10-12]$ and are presented in Table 1. The input files of the WinAct software package are created on the base of these results and compiled as shown in Figure 2.

From the output files, the leaves rate of a particular drug from the blood is estimated. This is an important indicator for minimizing dose loads on the body as a whole. In addition, the percentage of activity retention for the preparations of the pathological nidus and organs plays a major role in setting the restrictions for the magnitude of the input activity of the drug.

The main task is the comparison of drugs based on radionuclide ${ }^{177} \mathrm{Lu}$ and drugs that are currently used in medical practice for the treatment of bone metastases, such as ${ }^{153 \mathrm{Sm}-E D T M P .}$ As it is noted above, phosphonates form very stable compounds with radionuclides from a number of rare earth elements, such as ${ }^{177} \mathrm{Lu}$ and ${ }^{153} \mathrm{Sm}$. Based on this, the calculation takes into account that the solution is not more than a $1 \%$ free radionuclide. Using the data mentioned above, the source files for $153 \mathrm{Sm}$ are in combination with ethylene diamine tetramethylene phosphonate.

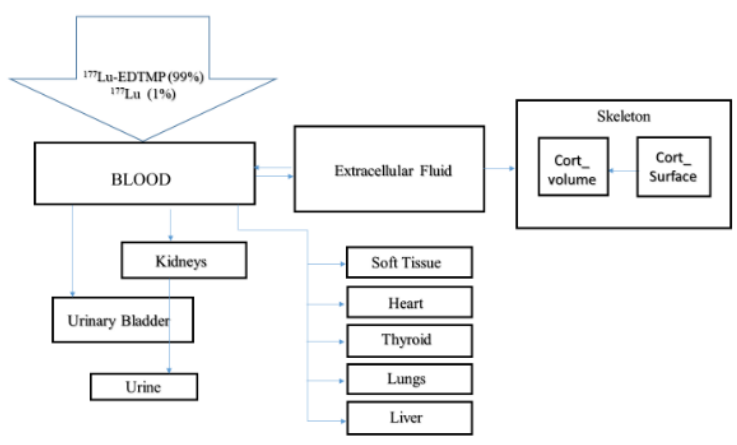

Figure 1. Biokinetic model of the drug ${ }^{177 \mathrm{Lu}-\mathrm{EDTMP}}$ or ${ }^{153 \mathrm{Sm}-}$ EDTMP taking into account the conversion

Table 1. Constant transition time between departments of osteotropic drug EDTMP

\begin{tabular}{|c|c|c|}
\hline \multicolumn{2}{|r|}{ Path } & \multirow{2}{*}{$\begin{array}{c}\text { Transition } \\
\text { Rate K, } \\
\text { Day }^{-1}\end{array}$} \\
\hline From & To & \\
\hline Blood & $->$ Ecf & 134 \\
\hline Ecf & -> Blood & 20.79 \\
\hline Ecf & -> Cort_Boone_Surf & 10.39 \\
\hline Ecf & -> Trab_Boon_Surf & 10.39 \\
\hline Blood & -> UB_Cont & 16.03 \\
\hline Blood & $->$ Soft Tissue & $3.0110^{-1}$ \\
\hline Blood & $->$ Spleen & $5.2010^{-2}$ \\
\hline Blood & -> Heart & $1.1010^{-2}$ \\
\hline Blood & $->$ Lungs & $3.8410^{-2}$ \\
\hline Blood & -> Liver & $9.0410^{-2}$ \\
\hline Blood & -> Kidneys & $1.1210^{-1}$ \\
\hline Soft Tissue & -> Blood & $1.4210^{-2}$ \\
\hline Spleen & $->$ Blood & $1.8010^{-2}$ \\
\hline Heart & $->$ Blood & $7.9510^{-2}$ \\
\hline Lungs & -> Blood & $2.2110^{-2}$ \\
\hline Liver & $->$ Blood & $7.3610^{-3}$ \\
\hline Kidneys & -> UB_Cont & $5.4710^{-2}$ \\
\hline
\end{tabular}

When using a drug based on radionuclide and a therapeutic agent, the dynamics of behavior in a body completely depends on the carrier. That is, the calculation files for the drug ${ }^{177 \mathrm{Lu}-E D T M P}$ and ${ }^{153 \mathrm{Sm}-}$ EDTMP are absolutely similar, except for the half-life of the radionuclide. In addition, in the process of further calculation the presence of the $1 \%$ free radionuclide in the solution will be taken into account, biokinetic data for which are not the same. A number of studies showed that the radiochemical resistance of drugs is not less than $99 \%$. Therefore, the free radionuclide ${ }^{177} \mathrm{Lu}$ in each solution will be taken into account separately in the calculations.

WinAct generates three output files and an information file with an extension ".log", which basically duplicates the input data. All files received as a result of the program are located in the \output folder. The file extension ".act" contains information on the activity contained in an organ or tissue as a function of the time since the beginning of the nuclide intake. It is this file that was used to plot the dependence of the activity retention on time after the administration of the drug. The file extension ".ext" contains data on the rate of excretion of the nuclide from urine and feces (1/day) 
as a function of time. Additionally, the file tabulates data on the retention of the nuclide in the lungs and in the body as a function of time. The file with the extension ".u50" contains data on the number of nuclear transformations in a particular organ or tissue.

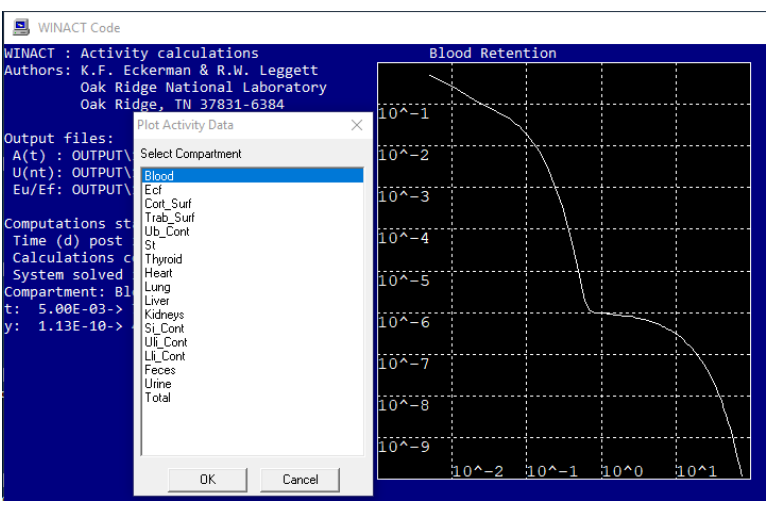

Figure 2. The input file of WinACt

The output results from the WinAct program are used as the input data for IDAC 2.1 software, an internal dosimetry program for nuclear medicine based on the ICRP adult reference voxel phantoms [13,14,15], IDAC 2.1 software was used for dose calculations in organs and tissues. As a result, the absorbed doses of the organs and tissues are estimated.

\section{RESULTS AND DISCUSSION}

To assess the level of radiation exposure risk to a patient due to radiopharmaceutical use, absorbed doses were calculated for the organs wherein the labeled complex accumulates to the greatest extent, namely, the kidneys, ECF, skeleton, spleen, heart, lungs, and liver. Since the ${ }^{177} \mathrm{Lu}$ and ${ }^{135} \mathrm{Sm}$ isotopes decay by emitting beta and gamma rays, it was also necessary to calculate the absorbed doses in the adjacent organs (targeted organ), which received radiation from the source organs. In connection to this, the doses in the lungs from the radionuclide in the liver, as well as the doses in the gonads from the contents of the bladder, were calculated as the most closely related organs. The injection was administered directly into the blood, which circulates throughout the body and to a large extent, in the lungs. The absorbed dose was assessed. Additionally, the contribution to the lungs, red bone marrow, and gonads from the source such as "other organs and tissues" was assessed. The results showed that for the rest of the internal organs, the contribution to the dose was smaller than for the listed organs by one or two orders of magnitude, so a detailed calculation of the doses was not performed for them.

Figures 3 and 4 refer to the time-activity curves of the radiopharmaceutical as fitted by WinAct. The figures show the behavior of fractional activity retention in different organs with ${ }^{177} \mathrm{Lu}$ and ${ }^{153 \mathrm{Sm}}$ in Ionic form and when labeled with EDTMP. The pharmaceuticals in ionic form were removed from the blood more slowly than when labeled with EDTMP, which points to the advantages of using ${ }^{177} \mathrm{Lu}-\mathrm{EDTMP}$ instead of ionic form. The largest amount of activity was cumulated in bone surface and a small amount was cumulated in other organs in the case of ${ }^{177 \mathrm{Lu}-E D T M P}$, which means that ${ }^{177} \mathrm{Lu}$-EDTMP is better than ${ }^{135 \mathrm{Sm}-E D T M P}$ in the diagnosis of bone metastases diseases. In addition, a long period of removal from the bones indicates that it is also better in therapy.
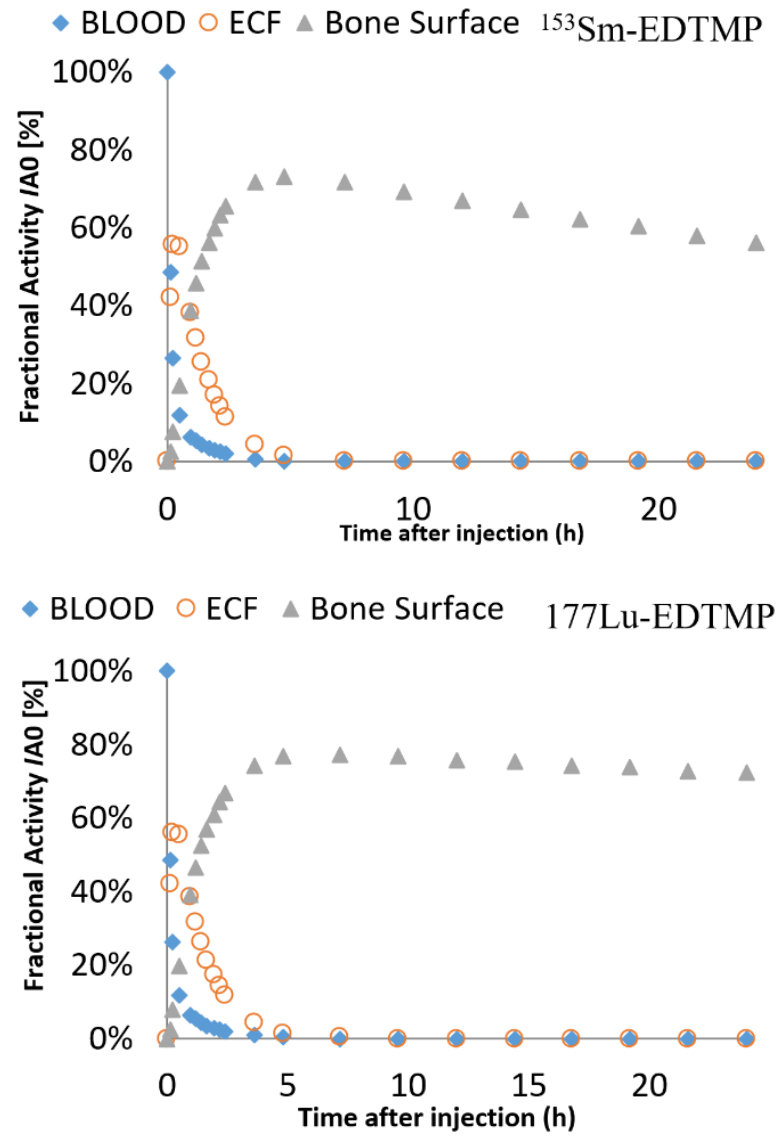

Figure 3. Distribution of activity in blood, ECF and bone surface of ${ }^{177 \mathrm{Lu}-E D T M P a n d}{ }^{153 \mathrm{Sm}-E D T M P}$

The peculiarity of this type of therapy is reasoned by the targeted action of the drug in the focus of the disease, a large dose is created and, very importantly, the dose load on healthy bone tissue, organs and tissues are minimized. The absorption in the bone tissue of a person with metastatic lesions of the skeleton depends on the degree of the disease, the general condition of the patient and many other factors. In the study, it is accepted that the absorption in the pathological area of bone tissue is $20 \%$ of the proportion of the substance deposited in a healthy skeleton.

Figure 5 shows the results of comparing a dose calculation conducted using the two drugs.

The ${ }^{177 \mathrm{Lu}-E D T M P}$ absorbed dose is two times higher than ${ }^{135} \mathrm{Sm}$-EDTMP with nearly the same effects on other organs. In addition, ${ }^{177 \mathrm{Lu}-E D T M P}$ like ${ }^{135} \mathrm{Sm}-$ EDTMP does not deposit in the liver unlike the ionic forms. The ionic form of ${ }^{177} \mathrm{Lu}$ has the absorbed dose two times lower than the ${ }^{135} \mathrm{Sm}$ ionic form in the liver with a high absorbed dose in the endosteum and red bone marrow. The highest effective dose per administered activity is $0.242 \mathrm{mGy} / \mathrm{MBq}$ for ${ }^{177 \mathrm{Lu}-E D T M P}$, followed

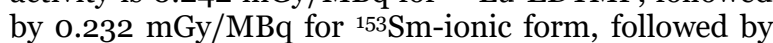
$0.189 \mathrm{mGy} / \mathrm{MBq}$ for ${ }^{177 \mathrm{Lu}-i o n i c}$ form and then 0.139 $\mathrm{mGy} / \mathrm{MBq}$ for ${ }^{153} \mathrm{sm}$-EDTMP. 

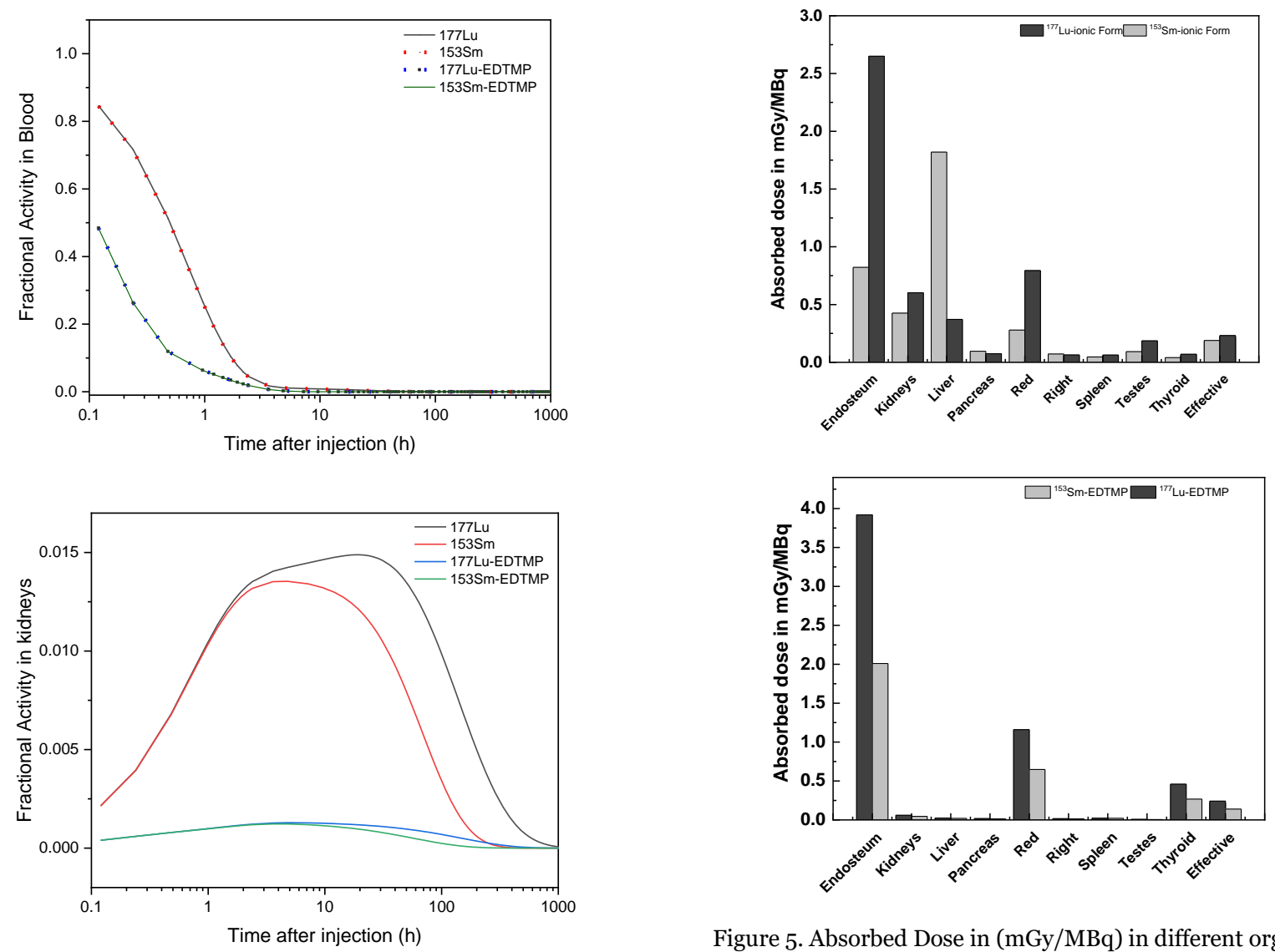

Figure 5. Absorbed Dose in (mGy/MBq) in different organs and tissues
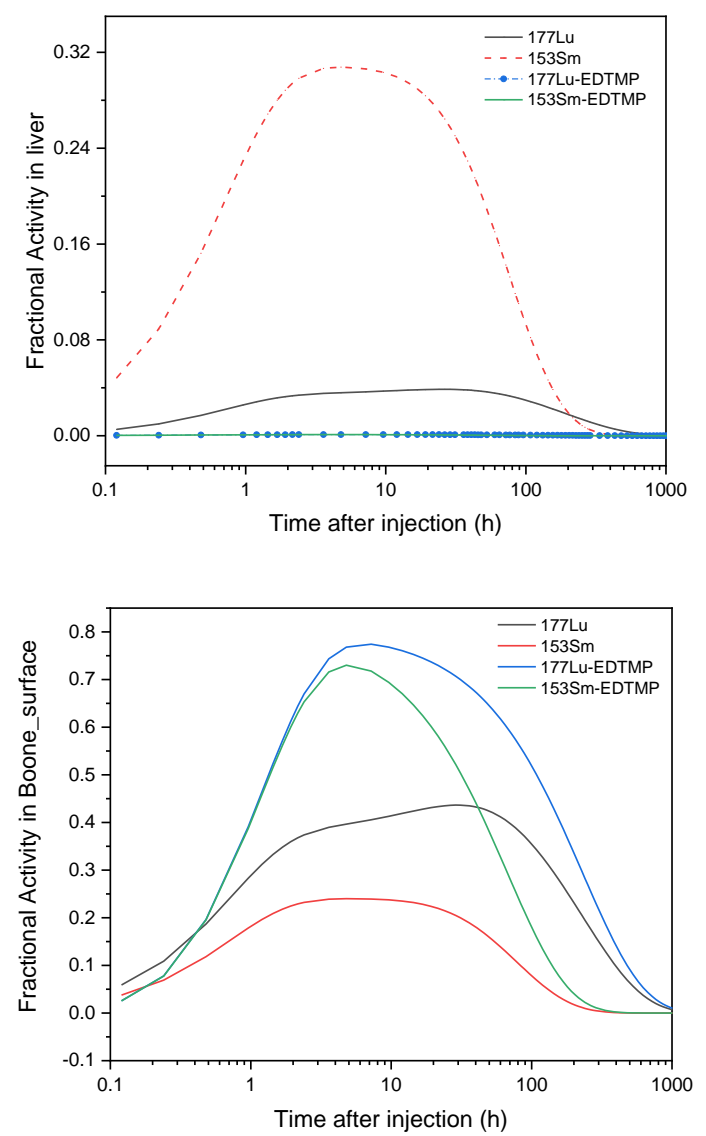

Figure 4. Fraction activities curves in blood and different organs for the three cases of interest

\section{REFERENCES}

1. S. Chakraborty et al., "177Lu-EDTMP: a viable bone pain palliative in skeletal metastasis," Cancer Biother. Radiopharm., vol. 23, no. 2, pp. 202 - 213, Apr. 2008. DOI: $10.1089 /$ cbr.2007.374 PMid: 18454689

2. P. Anderson, R. Nuñez, "Samarium lexidronam (153SmEDTMP): skeletal radiation for osteoblastic bone metastases and osteosarcoma," Expert Rev. Anticancer Ther., vol. 7, no. 11, pp. 1517 - 1527, Nov. 2007. DOI: $10.1586 / 14737140.7 .11 .1517$ PMid: 18020921 
3. A. Ahonen et al., "Samarium-153-EDTMP in bone metastases," J. Nucl. Biol. Med., vol. 38, suppl. 1, pp. 123 - 127, Dec. 1994

PMid: 7543288

4. I. G. Finlay, M. D. Mason, M. Shelley, "Radioisotopes for the palliation of metastatic bone cancer: a systematic review," Lancet Oncol., vol. 6, no. 6, pp. $392-400$, Jun. 2005.

DOI: 10.1016/S1470-2045(05)70206-0

PMid: 15925817

5. S. E. Abram, "Radiopharmaceutical Therapy for Palliation of Bone Pain from Osseous Metastases," in The Year book of anesthesiology and pain management, D. H. Chestnut, Eds., 1st ed., Maryland Heights (MO), USA: Mosby, 2006, pp. $256-257$ DOI: $10.1016 / \mathrm{s} 1073-5437(08) 70502-3$

6. T. Das, H. D. Sarma, A. Shinto, K. K. Kamaleshwaran S. Banerjee, "Formulation, preclinical evaluation, and preliminary clinical investigation of an in-house freezedried EDTMP kit suitable for the preparation of $177 \mathrm{Lu}-$ EDTMP," Cancer Biother. Radiopharm., vol. 29, no. 10 pp. $412-421$, Dec. 2004.

DOI: $10.1089 /$ cbr.2014.1664 PMid: 25409337

7. K. F. Eckerman, R.W. Leggett, WinAct version 1.O ORNL, Oak Ridge (TN), USA, 2002.

Retrieved from: https://www.ornl.gov/crpk/software Retrieved on: Mar. 22, 2019

8. H. M. H. Zakaly, M.Y.A. Mostafa, M. Zhukovsky, "Dosimetry Assessment of Injected 89Zr-Labeled Monoclonal Antibodies in Humans," Radiat. Res., vol. 191, no. 5, pp. 466 - 474, May 2019.

DOI: $10.1667 / R R 15321.1$ PMid: 30896281

9. M. Y. A. Mostafa, H. M. H. Zakaly, M. Zhukovsky, "Assessment of exposure after injection of $99 \mathrm{mTc}-$ labeled intact monoclonal antibodies and their fragments into humans," Radiol. Phys. Technol., vol. 12, no. 1, pp. 96 - 104, Mar. 2019.

DOI: $10.1007 / \mathrm{s} 12194-018-00496-1$

PMid: 30604358
10. S. Chakraborty, T. Das, H. D. Sarma, M. Venkatesh S. Banerjee, "Comparative studies of $177 \mathrm{Lu}$-EDTMP and $177 \mathrm{Lu}-\mathrm{DOTMP}$ as potential agents for palliative radiotherapy of bone metastasis," Appl. Radiat. Isot., vol. 66, no. 9, pp. 1196 - 1205, Sep. 2008.

DOI: 10.1016/j.apradiso.2008.02.061

PMid: 18372188

11. L. Vigna et al., "Characterization of the $[(153) \mathrm{Sm}] \mathrm{Sm}$ EDTMP pharmacokinetics and estimation of radiation absorbed dose on an individual basis," Phys. Med., vol. 27 , no. 3, pp. 144 - 152, Jul. 2011

DOI: 10.1016/j.ejmp.2010.08.001

PMid: 20864370

12. D. M. Taylor, R. W. Leggett, "A generic biokinetic mode for predicting the behaviour of the lanthanide elements in the human body," Radiat. Prot. Dosim., vol. 105, no. 1 - 4, pp. 193 - 198, 2003.

DOI: 10.1093/oxfordjournals.rpd.aoo6222 PMid: 14526955

13. M. Andersson, L. Johansson, K. Eckerman, S. Mattsson, "IDAC-Dose 2.1, an internal dosimetry program for diagnostic nuclear medicine based on the ICRP adult reference voxel phantoms," EJNMMI Res., vol. 7, no. 88, Nov. 2017.

DOI: $10.1186 / \mathrm{s} 13550-017-0339-3$

PMid: 29098485

PMCid: PMC5668221

14. H. M. H. Zakaly, M. Y.A. Mostafa, M. Zhukovsky, "Radiopharmaceutical dose distribution in different organs and tissues for Lu-177 with different carrier," AIP Conf. Proc., vol. 2174, no. 1, 2019.

DOI: $10.1063 / 1.5134421$

15. Education and Training in Radiological Protection for Diagnostic and Interventional Procedures, vol. 39, ICRP Publication no. 113, ICRP, Ottawa, Canada, 2009.

Retrieved from: https://journals.sagepub.com/doi/pdf/10.1177/ANIB 395

Retrieved on: May 8, 2019 